\title{
COMUNIDADES DE APRENDIZAJE A TRAVÉS DE PLATAFORMAS DE TELEFORMACIÓN
}

\author{
(LEARNING COMMUNITIES IN E-LEARNING PLATFORMS)
}

\author{
Ramón Tirado-Morueta \\ Ángel Hernando-Gómez \\ Ignacio Aguaded \\ Universidad de Huelva (España)
}

\section{RESUMEN}

En el marco del practicum de Educación Social, en la Universidad de Huelva se pone a prueba la plataforma WebCT como apoyo para la creación y desarrollo de comunidades de aprendizaje, creadas en función al ámbito profesional de prácticas, con la finalidad de resolver cooperativamente a través de foros casos reales presentados a los estudiantes. Utilizando como instrumento de recogida de datos el cuestionario, y como método de análisis el estudio de las frecuencias y el test de chi cuadrado se concluye que: a) la formación previa de los estudiantes en el uso de plataformas para el trabajo en equipo es un aspecto previo y fundamental para la creación de una comunidad de aprendizaje; b) la presencia del tutor es un factor asociado a la creación de un clima de confianza y apoyo, así como a la cooperación cognitiva entre compañeros; c) el sentimiento de comunidad de los estudiantes se asocia a la participación y compromiso de los estudiantes con el grupo y a la presencia social del tutor. Esta experiencia y los resultados que se presentan en este artículo sirvieron de impulso y referencia en sucesivas experiencias formativas y trabajos de investigación.

Palabras clave: E-learning, plataforma, prácticas, interacción, entornos de aprendizaje, comunidades virtuales.

\begin{abstract}
As part of the practicum for the Social Education diploma course at the University of Huelva (Spain), a WebCT platform was used in academic year 2007-08 to support creation and development of learning communities of students engaged in online discussions to resolve the real cases they faced at their work centres. Analysing the frequency distributions and correlations in data collected via a questionnaire, the following conclusions were drawn: (a) prior training of students in the use of learning management systems for cooperative learning is a fundamental aspect to the creation of a learning community; (b) the presence of the tutor is associated with creation of an environment of trust and support within the
\end{abstract}


community; (c) the sense of community among the students is associated with their participation in and commitment to the group and the social presence of the tutor. The results of this experiment serve as a point of reference for future practice and research.

Keywords: e-learning, learning management systems (LMS), practicals, interaction, learning environments, virtual communities.

Las prácticas curriculares son un momento especialmente significativo en la carrera académica de los estudiantes. Significan su primer contacto directo con la profesión después de años de trabajo intelectual, por lo que parece conveniente articular procesos de praxis que faciliten la convergencia entre conocimientos teóricos, situaciones prácticas y toma de decisiones.

Por otra parte, nos planteamos que esta riqueza de experiencias diversas de aprendizaje práctico e individual podríamos aprovecharla mejor si las compartimos entre todos los estudiantes del prácticum. Por estas razones, nos propusimos el uso didáctico de la plataforma formativa disponible para la Universidad de Huelva en el curso 2004-05 con el propósito de que nos sirviera de apoyo para la creación y desarrollo de una comunidad de aprendizaje entre los estudiantes del prácticum de la diplomatura de Educación Social.

Entender el concepto de comunidad de aprendizaje supone comprender que sus miembros son tanto estudiantes como profesores, lo que exige una obligada coordinación del profesorado entre sí, tanto en la distribución de grupos de estudiantes como en la aplicación de pautas comunes de acción tutorial, y una implicación profunda en un proyecto común. Esto ya en sí mismo supondría un reto difícil de superar en una primera experiencia como esta. Conscientes de ello, este proyecto se propuso, fundamentalmente, servir de experiencia piloto que aportase referencias para abordar con éxito próximos proyectos.

\section{REVISIÓN DE LITERATURA}

Ya hace años que las TIC se vienen usando para crear comunidades virtuales con el propósito de facilitar el aprendizaje colaborativo entre individuos que comparten un proyecto común pero se encuentran físicamente separados (Shea y Bidjerano, 2009; Aguaded y Cabero, 2002). Sin embargo, todas las comunidades de aprendizaje no resultan eficaces en el desarrollo de sus tareas, sin llegar apenas a ser algo más que un grupo de personas inscritos en un mismo curso y que disponen de un espacio en internet en el que sólo algunas de ellas se comunican. Con este trabajo tratamos 
de identificar algunos de los factores que influyen en la creación de verdaderas comunidades de aprendizaje.

Las comunidades de aprendizaje pueden hacer de puente entre la escuela y el entorno laboral. Solucionar de manera colaborativa problemas reales en una situación académica permite un campo de práctica realista y seguro que facilita el desarrollo de habilidades transferibles a situaciones de trabajo reales (Barab y Duffy, 2000). También facilita oportunidades para reflexionar, analizar y estudiar sobre casos reales, algo que en una situación de trabajo real es normalmente difícil. Esta es una de las principales razones que nos llevaron a plantear este proyecto. Las prácticas curriculares de los estudiantes universitarios son un periodo especialmente aprovechable para que se produzcan procesos de aprendizaje realmente significativos dado que se parte de problemas o casos reales que les plantea en su centro o programa de prácticas. Sin embargo, estas oportunidades generalmente no se aprovechan en todo su potencial dado que el estudiante suele actuar generalmente de modo casi intuitivo y de manera aislada del resto de sus compañeros, por lo que estas experiencias no se formalizan o exteriorizan para que puedan compartirse. El uso de las TICs y de las plataformas de teleformación nos permite superar este aislamiento en el que se encuentran los estudiantes durante sus periodos de prácticas, compartir experiencias y distribuir el aprendizaje partiendo del estudio de casos reales.

La expresión comunidad de aprendizaje se ha usado para describir aquella que incorpora una cultura de aprendizaje en la que cada miembro se implica en un esfuerzo colectivo para comprender los contenidos propuestos (Bielaczyc y Collins, 1999).

\section{Características de una comunidad de aprendizaje}

Las comunidades de aprendizaje comparten cualidades esenciales semejantes a la noción de comunidades de prácticas descrita por Wenger (1998, p. 73). Esencialmente, los participantes deben implicarse en un proyecto común, en el que las metas y proyectos se configuran entre todos; tienen un acceso común a un repertorio compartido de recursos, historias, conceptos y herramientas; y mantienen sus relaciones a través del compromiso, la confianza y la motivación mutua.

Basándonos en lo que sugieren estas definiciones, en las aportaciones de Wenger (1998) sobre comunidades de prácticas y en las revisiones de literatura sobre comunidades de aprendizaje realizada por Rovai (2002) y Ludwig-Hardman (2003) hemos identificado una serie de rasgos que nos ayudan a definirlas. Nosotros aquí vamos a centrarnos en las que se ajustan a los rasgos de este proyecto: 
- Sentimiento de comunidad. Reconocimiento de pertenencia al grupo, sentimiento de amistad, cohesión que se desarrolla entre los estudiantes. McMillan y Chavis (1986, p. 9) definían una comunidad como un sentimiento de pertenencia, un sentimiento compartido por cada uno de los componentes del grupo atendiendo a sus necesidades desde el compromiso de todos. Otros definen el sentido de comunidad como un resultado de la interacción y deliberación de un grupo de personas con intereses similares y metas comunes (Westheimer y Kahne, 1993), o como un ambiente en el que la gente interactúa de manera unida, reflexionan continuamente sobre el trabajo del grupo respetando siempre las diferencias individuales (Graves, 1992).

- Condiciones de apoyo y confianza. La participación de los estudiantes en el establecimiento de reglas de participación facilita el respeto y la confianza entre los estudiantes (Coombe, 1999; Retallick, 1999), lo que estimula el intercambio de ideas y un apoyo mutuo, que se comparta la responsabilidad sobre los resultados (Barab y Duffy, 1998; Downes, 1998; Hiltz, 1998; Jonasen, Peck y Wilson, 1999; Wells, 1999). Asimismo, es importante que el profesor asuma un rol respetuoso con las interacciones de grupo y haga seguimiento de las relaciones entre los estudiantes.

- Colaboración e interacción. La colaboración en un entorno de aprendizaje online suele ser un reto para los estudiantes. Condiciones tales como conseguir las contribuciones de cada uno a tiempo puede ser difícil, dependiendo del compromiso de los miembros del grupo y de ciertas preconcepciones que relacionan el aprendizaje online con el aprendizaje por correspondencia, individualizado o autónomo. Modelos débiles de colaboración como la asignación de tareas a cada miembro del grupo son más fáciles de sostener que modelos fuertes que requieren negociar, gestionar y tomar decisiones. Estas versiones fuertes de la colaboración serán una prueba de la coherencia del grupo, y a la vez fortalecerán los vínculos entre sus miembros y el sentimiento colectivo de comunidad.

Son varios los factores relativos a la presencia del profesor/tutor (Anderson, Rourke, Garrison, y Archer, 2001; Garrison, 2007; Garrison, Cleveland-Innes y Fung, 2004; Garrison, Cleveland-Innes, Koole y Kappelman, 2006; Arbaugh y Hwang, 2006; Zhu, 2006) en la gestión de la comunidad de aprendizaje, que tienen una relación positiva con la creación de una comunidad de aprendizaje. Atendiendo a las peculiaridades que definen este proyecto de comunidad de prácticas, nos centraremos en las siguientes: 
- Distancia transaccional. Moore (1993) define la distancia transaccional como el espacio de comunicación y psicológico entre los estudiantes y el tutor, siendo relativa y diferente para cada persona. De acuerdo con Moore, la extensión de la distancia transaccional depende de la estructura y el diálogo. La estructura es la cantidad de control que el tutor ejerce sobre el ambiente de aprendizaje, de manera que mientras más control ejerza el tutor sobre el grupo más aumentará la distancia psicológica y decrecerá el sentido de comunidad. El diálogo, por otra parte, es la cantidad de control ejercida por el estudiante, de modo que mientras mayor sea la cantidad de control ejercida por el estudiante la distancia psicológica decrecerá y aumentará el sentido de grupo.

- Presencia social del tutor/coordinador. Se trata de un factor que condiciona en buena medida el sentido de comunidad. El tutor tendrá que pensar las estrategias necesarias para hacer que aparezcan durante el desarrollo del curso indicios que hagan que los estudiantes no se sientan aislados.

- Facilitación del grupo. Los tutores deberán prestar atención a dos tareas fundamentales: las relacionadas con las tareas del grupo, y las relativas a la construcción, animación y mantenimiento del grupo. Para facilitar las interacciones relacionadas con la tarea, los tutores no deben olvidar que lo importante es que los estudiantes construyan por ellos mismos el conocimiento, de manera que deberán evitar todo protagonismo. Por otra parte, algunas de las funciones que ayudan a crear y mantener el sentido de grupo son las de animador, conciliador, moderador, organizador, observador o seguidor.

Si bien, el comentario de estos factores que en diferente medida modelan, condicionan y ayudan a definir la idea de comunidad de aprendizaje, existen modelos operativos que facilitan el abordaje empírico de la temática, basados en la definición de dimensiones diferenciadas y excluyentes entre sí (De Wever, Schellens, Valcke y Van Keer, 2006). En este sentido, uno de los modelos que más se ajustan al análisis de comunidades de aprendizaje en el contexto de la educación superior son los de Garrison, Anderson, y Archer (2000, 2001), Rourke, Anderson, Garrison, y Archer (1999) y Anderson et al., (2001). Aunque tales instrumentos se utilizan para el análisis de contenidos, nosotros lo utilizaremos como referencia para la selección de indicadores.

El instrumento creado por Rourke et al. (1999) se basa en el análisis de la presencia social que es uno de los tres elementos de una comunidad de aprendizaje tal y como es conceptualizada por Rourke et al. (1999). La presencia cognitiva y 
la presencia del tutor/profesor: "La presencia social potencia el logro de objetivos cognitivos a través de su capacidad de instigar, sostener y apoyar el pensamiento crítico en una comunidad de estudiantes" (Rourke et al., 1999, p. 54). Mensajes sociales tales como chistes, agradecimientos, saludos, alabanzas, suceden a menudo en las discusiones asincrónicas online y se consideran importantes para la motivación de los estudiantes.

Por otra parte, el instrumento de Garrison et al. (2001) se basa en el análisis de la presencia cognitiva, que es otro de los elementos del modelo de comunidad de aprendizaje. "La presencia cognitiva refleja la adquisicióny aplicación de conocimiento de orden superior, estando muy asociada con la literatura e investigación relativa al pensamiento crítico" (Garrison et al., 2001, p. 7).

La presencia del profesor/tutor (de enseñanza) (Anderson, Rourke, Garrison, y Archer, 2001) es el tercer elemento del modelo. Los autores ven la función del profesor seccionada en tres roles principales: primero, como diseñador de la experiencia educativa, incluyendo la planificación y administración de la instrucción; segundo, como facilitador y co-creador del ambiente social dirigido a activar y conseguir los objetivos; y finalmente, como experto en la materia que conoce mejor el tema que la mayoría de los estudiantes y actuando como facilitador de experiencias de aprendizaje, actuando como instructor.

\section{DESCRIPCIÓN DEL PROYECTO}

El proyecto de innovación nació con la intención de explotar los recursos de las plataformas de teleformación (Aguaded et al., 2010) para la creación de una comunidad de aprendizaje, permitiendo superar las limitaciones y aprovechar el potencial de las prácticas curriculares. El proyecto se articula en torno a tres ejes en respuesta a tres limitaciones frecuentes de este tipo de experiencias formativas:

- Mejora de la información y orientación del estudiante a través de un espacio informativo en el que se incorporarán datos como el plan de prácticas, el programa de prácticas, el calendario y un servicio de webmail para informar a los estudiantes de todas aquellas novedades que se fuesen produciendo.

- Mejora de la comunicación entre tutores y estudiantes en periodo de prácticas, aprovechando el potencial de las diversas aplicaciones de internet para mantener una comunicación fluida entre los tutores y los estudiantes de prácticas, superando las incompatibilidades horarias y las posibles dificultades de desplazamiento. 
- Mejora de la comunicación y fomento del debate entre los estudiantes sobre los casos prácticos con los que se están enfrentando. Se trata de usar los foros de discusión para compartir y debatir las experiencias prácticas de los estudiantes a través de la articulación teórico-práctica de las situaciones y problemas reales a los que se enfrentaban (Tirado, 2002, 2003, 2005).

Para el desarrollo del proyecto contamos con la plataforma WebCT de la Universidad de Huelva, durante el curso 2004-05. Entre otros elementos, en este espacio organizamos tres tipos de foros: uno general, otro de discusión dirigido sobre casos prácticos, y otro para la solución de dudas o dificultades del estudiante.

- El foro general está abierto para todos los estudiantes. Este foro tenía como objetivo servir de lugar de encuentro para todos los estudiantes en prácticas.

- El foro de dudas o dificultades pretendía canalizar las dudas o dificultades de los estudiantes con el propósito de facilitar el acceso de los alumnos a su tutor sin los condicionantes de espacio y tiempo, permitiendo que los estudiantes sean atendidos sin necesidad de desplazarse a su despacho y en su horario de tutoría. Si nos decidimos por centrar todas las dudas de los estudiantes en un único foro fue para que cualquier estudiante o profesor pueda intervenir y responder según sus recursos o conocimientos, pensando en que el potencial de respuesta del grupo general es superior al de un tutor particular asignado a un alumno.

- Foro de casos: Se trata de aprovechar las virtudes de esta herramienta telemática para formalizar los conocimientos prácticos que se vayan adquiriendo durante las prácticas, desde un proceso de reflexión colaborativa sobre casos reales. En este foro el estudiante planteaba una situación profesional real que se hubiese presentado y obligado a tomar una decisión. Los demás compañeros tratarían de documentarse, aportar posibles soluciones al caso esgrimiendo argumentos. Finalmente, el mismo alumno que ha expuesto el caso, considerando las aportaciones de sus compañeros y su propia experiencia elaboraría una resolución precisa del caso.

De este modo los conocimientos teóricos adquiridos y las capacidades analíticas de los estudiantes comienzan a movilizarse y a tomar sentido en circunstancias reales. El conocimiento tácito se exterioriza, formaliza y comparte (Tirado, 2002), sirviendo como base de conocimiento para futuras promociones de alumnos en prácticas. El resultado será una serie de estudios de casos reales que pueden ser usados en las clases convencionales como hilo conductor de ciertos contenidos curriculares. 
R. Tirado; Á. Hernando; I. AguAded

Comunidades de Aprendizaje a Través de Plataformas de Teleformación

\section{OBJETIVOS}

A través de esta experiencia tratamos de explorar las posibilidades de las plataformas digitales para crear comunidades de aprendizaje. Los objetivos que nos planteamos en esta investigación fueron los siguientes:

- Averiguar en qué medida el uso de una plataforma de teleformación ha permitido satisfacer las expectativas de los estudiantes al utilizar este medio de información y comunicación en un contexto de prácticas curriculares universitarias.

- Indagar en la relación que la capacitación tecnológica de los estudiantes ha tenido sobre el desarrollo de la comunidad de aprendizaje en dos de sus dimensiones que la definen: presencia social y presencia cognitiva.

- Corroborar la relación que mantiene la presencia del tutor/profesor y la organización de los recursos en la plataforma con la creación de una comunidad de aprendizaje.

\section{MÉTODO}

Metodológicamente en este estudio usamos un enfoque cuasiexperimental basado en un diseño tipo ATI, de investigación en medios, para lo cual hemos utilizado dos cuestionarios, uno que aplicamos antes de la experiencia y otro al finalizarla. El primero de ellos tenía la finalidad de conocer las expectativas de los estudiantes sobre el uso de la plataforma como espacio de comunicación durante las prácticas curriculares. El cuestionario final tiene el objetivo de conocer el grado en el que se han satisfecho tales expectativas, así como de recoger las valoraciones de los condicionantes de enseñanza (presencia del tutor/profesor) y mediáticos para el desarrollo de comunidades de aprendizaje.

Para la construcción de los cuestionarios hemos atendido a las dimensiones que comentamos a continuación. En primer lugar, la alfabetización tecnológica de los estudiantes. Las habilidades comunicativas y competencias tecnológicas previas que disponen los alumnos determinan y condicionan el proceso de aprendizaje (Meneses, González y Fandos, 2005). En segundo lugar, hemos considerado las tres categorías que definen el modelo de comunidad de aprendizaje elaborado por Garrison et al. (2000, 2001), así como los recursos que pueden condicionar la creación de una comunidad de aprendizaje (Rovai, 2002). Para la creación de indicadores hemos atendido a las definiciones de las dimensiones que realizan los autores del modelo. 
Por tanto, tales indicadores son susceptibles de ser validados en estudios futuros. Cada uno de los indicadores ofrece seis modalidades de respuesta atendiendo a una escala tipo Likert con valores comprendidos entre o y 5 , permitiendo que el alumno exprese el grado de valoración de cada ítem o afirmación.

\begin{tabular}{|c|c|}
\hline Dimensiones & Indicadores \\
\hline $\begin{array}{c}\text { Presencia Social } \\
\text { (Condiciones de apoyo y } \\
\text { confianza/Sentimiento de } \\
\text { comunidad) }\end{array}$ & $\begin{array}{l}\text { Mantener el contacto afectivo con mis compañeros de curso } \\
\text { Charlar con mis compañeros } \\
\text { Mantenerme informado de las novedades del prácticum } \\
\text { Expresarme libremente sin que nadie me interrumpa } \\
\text { Comunicarme a cualquier hora del día o de la noche } \\
\text { He contado aquello que no me atrevería a decir cara a cara } \\
\text { Participar abiertamente en todos los temas debatidos } \\
\text { Me he comunicado mejor con mi tutor } \\
\text { Familiarizarme con el uso de plataformas de comunicación } \\
\text { Informar y que los compañeros me informen de cursos que se ofertan } \\
\text { Seguir informado de novedades laborales que pudieran interesarme }\end{array}$ \\
\hline $\begin{array}{l}\text { Presencia Cognitiva } \\
\text { (Colaboración) }\end{array}$ & $\begin{array}{l}\text { Argumentar sin límites de tiempo mis ideas } \\
\text { Compartir y debatir las experiencias de los demás compañeros } \\
\text { Mis compañeros me han ayudado a solucionar dudas que me surgieron } \\
\text { He encontrado recursos que me han ayudado a realizar trabajos } \\
\text { Tener una experiencia comunicativo-formativa novedosa }\end{array}$ \\
\hline Presencia del tutor & $\begin{array}{l}\text { Nivel de respuesta de los tutores a nuestros comentarios } \\
\text { Rapidez de respuesta de los tutores a las dudas planteadas en los foros } \\
\text { La animación para participar en los foros }\end{array}$ \\
\hline Recursos & $\begin{array}{l}\text { Número de foros en la plataforma } \\
\text { Facilidad de respuesta y lectura de los comentarios en los foros } \\
\text { Claridad en la temática de cada foro } \\
\text { Facilidad de manejo de la plataforma } \\
\text { El acceso a la plataforma desde mi centro de prácticas } \\
\text { Cada foro se ha usado exclusivamente para lo previsto }\end{array}$ \\
\hline
\end{tabular}

Cuadro 1. Indicadores de comunidad de aprendizaje incluidos en los cuestionarios

Para comprobar la fiabilidad de los instrumentos aplicamos la prueba Alfa de Crombach a todos los ítems obteniendo 0,72 en la serie de cuestiones relativas al nivel de formación y de 0,93 en la serie de cuestiones dedicadas a Presencia Social, Presencia cognitiva, Presencia del tutor y Recursos. El cuestionario completo supera la puntuación de o,93 en esta prueba, por lo que podemos pensar que el instrumento tiene un elevado nivel de fiabilidad. Estos instrumentos se aplicaron a la totalidad 
de alumnos que asistieron a una jornada previa al uso de la plataforma y a la de evaluación final del prácticum. Para el análisis de los datos se utilizó el programa SPSS 15.0.

\begin{tabular}{|l|l|r|r|r|r|r|r|}
\cline { 3 - 8 } \multicolumn{2}{|c|}{} & $\begin{array}{c}\text { Frecuencia } \\
\text { inicio }\end{array}$ & $\begin{array}{c}\text { Porcentaje } \\
\text { inicio }\end{array}$ & $\begin{array}{c}\text { Porcentaje } \\
\text { válido inicio }\end{array}$ & $\begin{array}{l}\text { Frecuencia } \\
\text { final }\end{array}$ & $\begin{array}{c}\text { Porcentaje } \\
\text { final }\end{array}$ & $\begin{array}{l}\text { Porcentaje } \\
\text { válido final }\end{array}$ \\
\hline Válidos & Total & 37 & 97.4 & 100.0 & 58 & 98.3 & 100.0 \\
\hline Perdidos & Sistema & 1 & 2.6 & & 1 & 1.7 & \\
\hline Total & 38 & 100.0 & & 59 & 100.0 & \\
\hline
\end{tabular}

Tabla 1. Frecuencia de respuesta (encuesta inicio y final)

Hemos agrupado las edades en tres grupos. Tenemos a los menores de 21 años que se correspondería a aquellos estudiantes de educación social que han seguido un itinerario limpio, es decir, que nunca han suspendido ni han repetido ningún curso. Los de 22 a 24 años es un grupo heterogéneo donde encontramos alumnos que han terminado otras carreras y continúan con educación social, o han cambiado de carrera, o han suspendido en cursos anteriores. Y los de más de 25 años que se corresponde a situaciones de alumnos que compatibilizan el trabajo y el estudio, o que terminaron de estudiar hace tiempo y al aparecer esta carrera han vuelto a retomar los estudios universitarios; los menos son los que han hecho el acceso a la universidad para mayores de 25 años (hemos conocido un par de casos que superan los 30 años).

\section{RESULTADOS}

Objetivo 1. ¿En qué medida el uso de una plataforma de teleformación ha permitido satisfacer las expectativas de los estudiantes al utilizar este medio de información y comunicación en un contexto de prácticas curriculares universitarias?

Nuestro primer propósito en esta investigación fue conocer qué esperaban los estudiantes de esta experiencia de comunidad de aprendizaje y comprobar si tales expectativas fueron satisfechas. El grado de satisfacción de sus expectativas sería un indicador del grado de desarrollo de la comunidad de aprendizaje. Veamos en que medida se satisficieron cada uno de los rasgos que definen el modelo de comunidad 
de aprendizaje que nos habíamos propuesto constituir:

- El principal propósito de la mayoría de los estudiantes, a priori, era seguir informado de las novedades laborales que pudieran producirse en el transcurso del prácticum. Debemos considerar que un gran número de los estudiantes no habían tenido experiencia previa con plataformas de formación, la mayoría de ellos usaban internet para charlar e intercambiar información, especialmente sobre lo que en esta etapa de sus carreras más les preocupa, es decir, sobre las vías de acceso al empleo. Respecto a lo que la experiencia supuso para los estudiantes encontramos que sirvió para que continuasen informados sobre cursos y otras vías de acceso al empleo.

- Mantenerse informado de las novedades del prácticum, seguir informado de novedades laborales, conseguir recursos, familiarizarse con el uso de plataformas y comunicarse fluidamente con su tutor fueron las expectativas más valoradas por el mayor número de estudiantes. Hemos comprobado que cuatro de estas expectativas, en general, se han satisfecho en buena medida para un buen número de estudiantes: mantenerse informado de las novedades del prácticum, mantener contacto afectivo con los compañeros, recibir ayuda al compartir experiencias y avanzar en el uso de plataformas.

- Uno de los principales objetivos del proyecto, tener una comunicación fluida con el tutor, no se ha satisfecho como esperábamos. Una de las principales razones que explican la decepcionante valoración que los estudiantes realizaron de la tutoría puede residir en la escasa formación específica previa que tuvo el equipo docente, unido a la opción de un foro único de dudas en el que cualquier tutor podría intervenir. Esto hizo que siempre fueran los mismos tutores los que participaran dado que las dudas que se planteaban no iban dirigidas a un tutor particular. Esto nos lleva a la conclusión de que en la próxima edición se plantee un foro de tutoría para cada tutor, de este modo esperamos que los tutores se impliquen más en las acciones de tutoría al tener su propio foro.

- Tener una experiencia formativa novedosa y que los compañeros respondiesen a las dudas o dificultades que surgiesen fueron las expectativas manifestadas por la mayor parte de los estudiantes. Probablemente esto sea debido a la falta de hábito en el uso de plataformas para la formación. De hecho, más adelante comprobaremos que la formación de los estudiantes se relaciona positivamente con este tipo de uso. Pues bien, no podemos afirmar que tales expectativas se hayan satisfecho como esperábamos. Gran parte de los estudiantes no se manifiestan satisfechos con las respuestas de sus compañeros ni con el contacto mantenido con ellos. 
Por tanto, podemos concluir que nos hemos acercado a la creación de una comunidad de aprendizaje, si bien quedan muchos aspectos que mejorar, fundamentalmente aquellos relativos a la presencia del profesor/tutor como son la implicación de los tutores en el uso de la plataforma y contacto con sus estudiantes, así como ofrecer un mejor repertorio de recursos útiles para la solución de problemas durante las prácticas. La creación de comunidades de aprendizaje hay que entenderla como un proceso de alfabetización progresivo que exige la implicación de todos sus miembros. En este sentido, debemos contar en lo sucesivo con la circunstancia de que en nuestra universidad, como suele ser normal en una universidad de tipo presencial, no existe el hábito de aprender a distancia de manera regular, lo que exige planificar periodos preparatorios para los estudiantes y profesores antes de

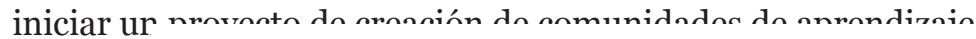

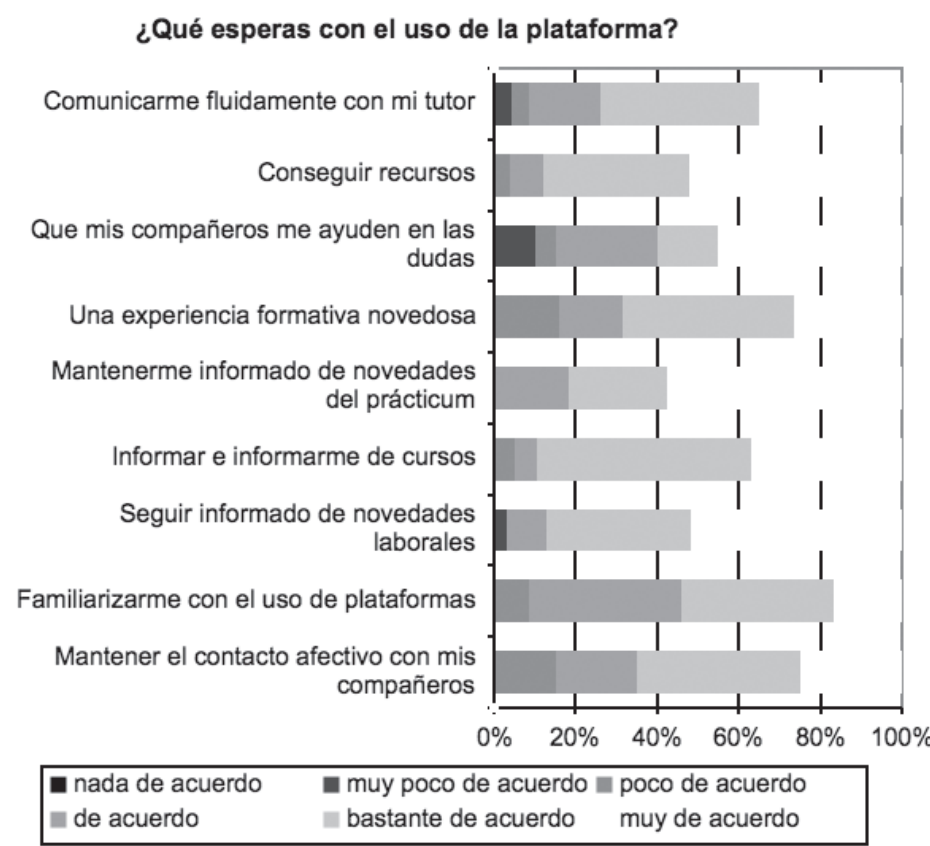




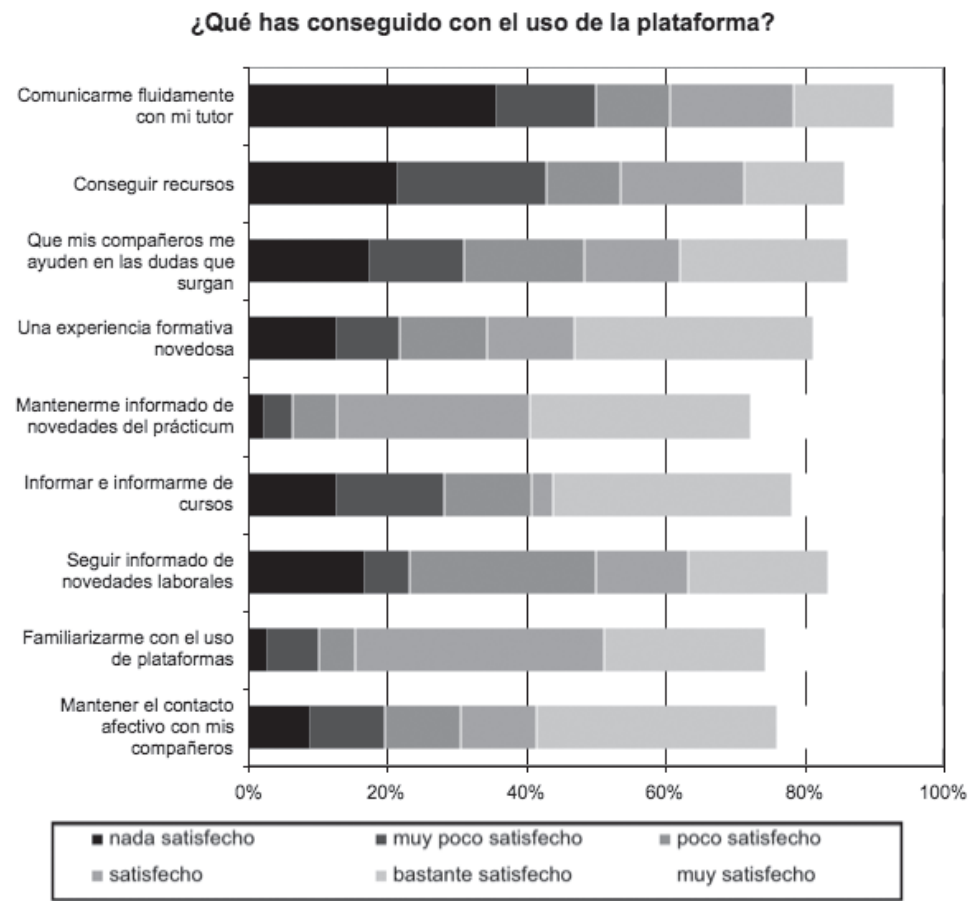

Gráfico 1. Expectativas sobre el uso de la plataforma Gráfico 2. Satisfacción con el uso de la plataforma

Objetivo 2. Indagar en la relación que la capacitación tecnológica de los estudiantes ha tenido sobre el desarrollo de la comunidad de aprendizaje en dos de sus dimensiones que la definen: presencia social y presencia cognitiva.

Para el análisis de la capacitación tecnológica de los estudiantes hemos diferenciado cinco dimensiones que consideramos fundamentales para el aprendizaje y generación de conocimientos a través de Internet (Tabla 2).

\begin{tabular}{|l|r|r|r|r|r|}
\cline { 2 - 7 } \multicolumn{1}{c|}{} & $\begin{array}{c}\text { Búsqueda y } \\
\text { organización de } \\
\text { información }\end{array}$ & $\begin{array}{c}\text { Chatear y } \\
\text { comunicarte con } \\
\text { otras personas }\end{array}$ & $\begin{array}{c}\text { Trabajo en } \\
\text { grupo a través } \\
\text { de Internet }\end{array}$ & $\begin{array}{c}\text { Diseño } \\
\text { Web }\end{array}$ & $\begin{array}{c}\text { Uso de } \\
\text { plataformas } \\
\text { formativas }\end{array}$ \\
\hline Válidos encuesta inicio & 38 & 38 & 38 & 37 & 37 \\
\hline Mediana encuesta inicio & 4 & 4 & 2 & 2 & 2 \\
\hline Válidos encuesta final & 58 & 58 & 58 & 58 & 58 \\
\hline Mediana encuesta final & 4 & 4 & 3 & 3 & 3 \\
\hline
\end{tabular}


Tabla 2. Grado de formación tecnológica de los alumnos antes y después de la experiencia

Digamos en primer lugar que uno de los resultados tras el análisis del valor de la mediana, nos lleva a la conclusión de que la experiencia formativa ha producido un incremento en el nivel formativo de los estudiantes respecto al trabajo en grupo a través de Internet, al diseño de web y al uso de plataformas.

Con la intención de corroborar la influencia de la capacitación tecnológica (Condi y Livingston, 2007; Tirado, Pérez y Aguaded, 2011) sobre el proceso de comunidad de aprendizaje, realizamos un análisis del valor de Chi cuadrado, tras el cual encontramos los siguientes datos (Tabla 3).

\begin{tabular}{|c|c|c|c|c|c|c|}
\hline & & \\
\hline & & 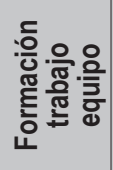 & 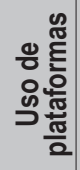 & 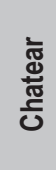 & 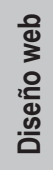 & 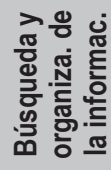 \\
\hline \multirow{4}{*}{ 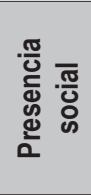 } & Mantener el contacto afectivo con mis compañeros de curso & & $* * *$ & ** & & \\
\hline & Mantenerme informado de las novedades del prácticum & & & $* * *$ & & \\
\hline & Expresarme libremente sin que nadie me interrumpa & & ** & & & \\
\hline & Participar abiertamente en todos los temas debatidos & *** & $* * *$ & & & \\
\hline \multirow{3}{*}{ 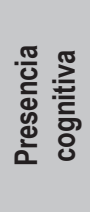 } & Argumentar sin límites de tiempo mis ideas & * & & *** & & \\
\hline & $\begin{array}{l}\text { Compartir y debatir las experiencias de los demás } \\
\text { compañeros }\end{array}$ & ** & *** & $* * *$ & *** & *** \\
\hline & $\begin{array}{l}\text { Mis compañeros me han ayudado a solucionar dudas que } \\
\text { me surgieron }\end{array}$ & ** & *** & & & \\
\hline
\end{tabular}

Tabla 3. Análisis Chi cuadrado entre el grado de formación de los estudiantes y el aprovechamiento $\left({ }^{*}=p<0,05 ;{ }^{* *}=p<0,01 ;{ }^{* * *}=p<0,001\right)$

En primer lugar encontramos que habilidades desarrolladas por los estudiantes, como el trabajo en equipo a través de Internet, el uso de plataformas y experiencia en el uso del Chat, se han mostrado como factores asociados positivamente con la participación abierta en los temas debatidos, la argumentación sin límite de los comentarios, así como sobre el debate de las experiencias, la colaboración y la ayuda entre compañeros.

El grado de formación y experiencia en el trabajo en equipo a través de Internet es un factor también asociado positivamente con el hecho de que el estudiante participe más en los temas debatidos, articule mejor sus argumentos y aportaciones, comparta experiencias y las discuta, y, en consecuencia, reciba más ayuda de los compañeros. 
La costumbre y habilidad en el uso del Chat también se revela como un factor asociado con el aprovechamiento de los foros como espacio de aprendizaje, si bien, se relaciona especialmente con la dimensión afectiva de la comunicación. Los estudiantes que hacen uso del Chat han encontrado en el proyecto una experiencia novedosa y les ha servido para mantener o establecer lazos afectivos con sus compañeros a través del foro abierto o general del prácticum. Además, los estudiantes que suelen usar el Chat se han mantenido informados de todas las novedades del prácticum. También hemos encontrando que estos estudiantes han argumentado ideas y compartido experiencias participando en los debates. Por tanto, parece que la costumbre del uso de Internet para comunicarse y expresarse es un factor que facilita el aprendizaje colaborativo a través de la red.

Compartir y debatir experiencias es un aspecto relacionado con todas aquellas competencias que hemos considerado para definir la alfabetización tecnológica: el uso de chat, el diseño de web, el conocimiento de plataformas, la búsqueda de información y el trabajo en grupo a través de Internet.

Objetivo 3: Corroborar la relación que mantiene la presencia del tutor/profesor y la organización de los recursos en la plataforma con la creación de una comunidad de aprendizaje.

Por último, partiendo del conocimiento de los factores que desde la literatura especializada se consideran asociados al proceso de configuración de una comunidad de aprendizaje (Rovai, 2002; Anderson et al., 2001; Garrison et al., 2000; 2001; Garrison y Archer, 2003; Garrison et al., 2006) y tras el análisis del valor de Chi cuadrado hemos encontrado las siguientes relaciones que nos ayudan a entender el sentido de la relación de estos factores con las diferentes dimensiones que ayudan a definir a una comunidad de aprendizaje (Tabla 4):

- La frecuencia y la rapidez de respuesta de los tutores. Este aspecto lo encontramos relacionado principalmente con el contacto fluido de estudiantes y tutores. La rapidez de respuesta del tutor también hace que los estudiantes lo consideren un mediador para conseguir recursos útiles en sus prácticas, se sientan más libres para manifestar sus pensamientos y se sientan informados de las novedades que puedan surgir en la comunidad. Por otra parte, la tutoría a través de la plataforma, en los casos en los que el tutor se ha implicado, se ha definido no solo como una mejora general de la comunicación, sino también como una fuente de recursos para el estudiante. Los estudiantes que manifiestan haber mejorado su comunicación 
con su tutor son aquellos cuyos tutores les han respondido con rapidez.

- Animación para participar en foros. La animación de los foros por parte de los tutores resulta un factor asociado a la interacción y la colaboración entre compañeros. También parece ser un factor que facilita aspectos como el conocimiento de la plataforma así como la expresión libre de los estudiantes.

- Organización de los recursos. El repertorio de recursos integrados en la plataforma se ha mostrado como un factor que en general se relaciona tanto con aspectos sociales, como cognitivos.

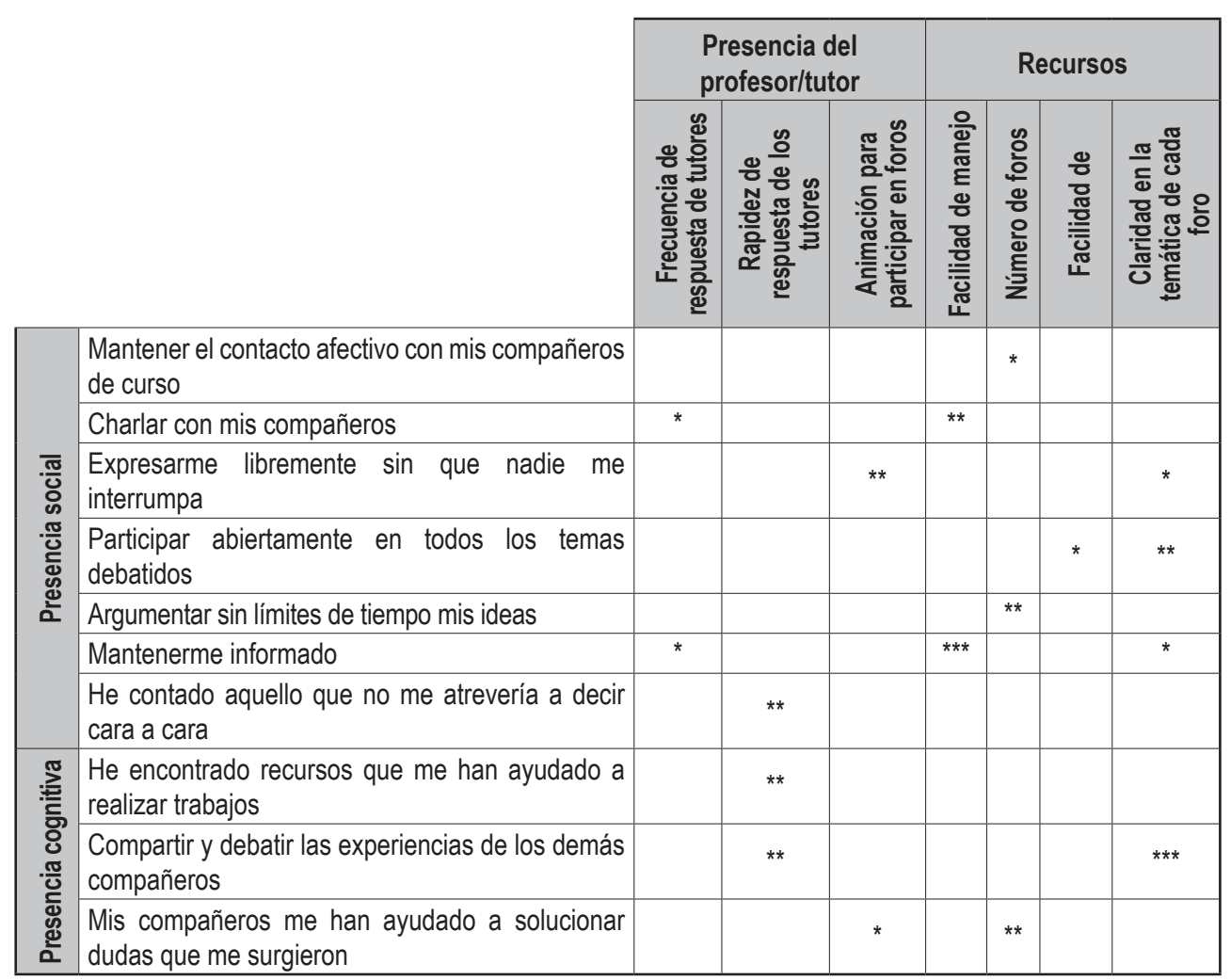

Tabla 4. Análisis Chi cuadrado entre la presencia del tutor/recursos y el aprovechamiento $\left({ }^{*}=p<0,05\right.$;

$$
\left.{ }^{* * *}=p<0,01 ;{ }^{* * *}=p<0,001\right)
$$

\section{CONCLUSIONES}

El objetivo del proyecto era provocar un proceso de creación de una comunidad 
R. Tirado; Á. Hernando; I. AguAdED

Comunidades de Aprendizaje a Través de Plataformas de Teleformación

de aprendizaje que permitiera compartir y profundizar en todo el conocimiento generado a partir de las experiencias que cada estudiante de manera particular viene teniendo en sus centros o programas de prácticas. Este objetivo nos llevó al concepto de comunidad de aprendizaje: que sus miembros asuman un sentimiento de comunidad, que se den las oportunas condiciones de apoyo y confianza, para que finalmente, se produzca interacción y colaboración entre todos los componentes de la comunidad, lo que llevaría a la comunidad a una situación de enriquecimiento mutuo y aprendizaje. Sin embargo, no podemos presumir de haber satisfecho nuestras expectativas en la medida deseada. Por suerte, este proceso de investigación nos ha ayudado a detectar algunas claves que nos pueden aportar sugerencias de mejora para venideras ediciones del proyecto.

- Respecto a la presencia del profesor/tutor. La presencia social del tutor se ha visto como un factor determinante para que se cree un clima de confianza y apoyo en la comunidad. Se requiere una mayor implicación del profesorado tanto en cuanto a su frecuencia de respuesta como a la rapidez de la misma, si no es así se corre el riesgo de que los estudiantes se desmotiven. Para lograr esta mayor participación de los tutores se podría utilizar un foro de tutoría para cada tutor. Esta medida esperamos que obligue al tutor a consultar su foro y atender a los estudiantes dado que la gestión del foro deja de ser anónima. Por otra parte, cada tutor deberá responder regularmente y con rapidez a las cuestiones que le planteen los estudiantes, lo que supone incluir como rutina docente la consulta diaria de la plataforma, si no lo hacemos así es muy probable que los estudiantes se sientan aislados. Otro factor asociado con el papel del tutor es la facilitación de la participación de los estudiantes en los foros o grupos de trabajo, esto es una condición especialmente importante cuando la comunidad se encuentra aún en un estado inmaduro.

- Respecto a la presencia social y cognitiva. Tal como ha quedado revelado a través de este primer estudio, la interacción y colaboración entre compañeros depende de múltiples factores como la participación o implicación de los compañeros, la respuesta de los tutores, la animación de los foros y del diseño de los elementos que definen el entorno de recursos en la plataforma. Como vemos son muchas las variables que hay que controlar para que se produzca uno de los principales fines del proyecto que no es otro que el intercambio y debate abierto y creativo sobre experiencias de aprendizaje durante situaciones de prácticas. A pesar de la dificultad que supone inferir implicaciones prácticas concluyentes en función de los resultados de esta investigación, sugerimos que se organicen grupos de trabajo más reducidos, a ser posible dinamizados cada 
uno por un profesor o tutor que se ocupe de la creación del clima de grupo, de la organización y protocolo del trabajo de equipo, de la coordinación en las diferentes fases del trabajo y, en los casos requeridos, de la instrucción sobre el tema o problema abordado.

- La ausencia de formación de los estudiantes en el trabajo en equipo a través de Internet nos hace sugerir la conveniencia de plantear protocolos de trabajo en equipo que les aporte alguna orientación respecto a como abordar las actividades propuestas al grupo. Si bien, en el caso de grupos inmaduros resultará conveniente una mayor presencia del tutor, especialmente, en las primeras fases del proceso, atendiendo y cuidando la distancia transaccional adecuada para la constitución de una comunidad de aprendizaje.

\section{IMPLICACIONES SOBRE PROYECTOS SUCESIVOS}

La experiencia y proyecto pionero que hemos expuesto en este artículo tuvo implicaciones pedagógicas y académicas ilustradas en múltiples trabajos publicados. A saber:

Proyectos de creación de comunidades virtuales de aprendizaje en periodos de prácticas. En sucesivos cursos académicos 2006-07, 2007-08, 2008-09, 2009-10 y 2010-11 se organizaron con la ayuda de plataformas (Moodle y WebCT) comunidades de aprendizaje en un mismo grupo académico atendiendo a temáticas diferenciadas según el ámbito de prácticas. De esta forma se organizan foros para cada grupo en orden al campo de prácticas, en los que se exponen casos prácticos reales a abordar entre compañeros distribuidos en distintos centros de trabajo (Tirado, Marín y Lojo, 2008; Tirado, Boza y Guzmán, 2009).

Seha estudiado los procesos de creación del conocimiento a través deinstrumentos para el análisis de contenidos, encontrando diferentes fases en el trabajo en equipo a distancia en grupos de comunicación asincrónica no estructurados.

Se ha analizado a través del Análisis de Redes Sociales (ARS) la estructura de las comunidades, cohesión, centralidad y centralización, y sus efectos sobre la creación social del conocimiento (Tirado, Hernando y Aguaded, 2011).

Se han desarrollado experiencias interuniversitarias e internacionales de teleformación basadas en la creación de pequeñas comunidades de aprendizaje entre compañeros de diferentes universidades, en España y Portugal (Tirado, Aguaded y 
R. Tirado; Á. Hernando; I. AguAdED

Comunidades de Aprendizaje a Través de Plataformas de Teleformación

Méndez, 2009; Tirado y Méndez, 2010).

Esta experiencia pionera ha sido uno de los gérmenes que ha dado origen a una línea de investigación y programas formativos que están hoy plenamente consolidados en la Universidad de Huelva, e internacionalmente en la educación superior.

REFERENCIAS BIBLIOGRÁFICAS

Aguaded, J. I.; et al. (2010). Plataformas de teleformación en las Universidades andaluzas. Huelva: Universidad de Huelva.

Aguaded, J. I.; Cabero, J. (Eds.) (2002). Educar en Red. Internet como recurso para la educación. Málaga: Aljibe.

Arbaugh, J. B.; Hwang, A. (2006). Does «teaching presence» exist in online MBA courses? The Internet and Higher Education, 9 (1), (9-21).

Anderson, T.; Rourke, L.; Garrison, D. R.; Archer, W. (2001). Assessing teaching presence in a computer conference context. Journal of Asynchronous Learning Networks. [en línea] Disponible en: http://www.sloan-c.org/ publications/jaln/v5n2/pdf/v5n2 anderson.pdf (consulta 2011, 18 de enero).

Barab, S. A.; Duffy, T. M. (2000). From practice fields to communities of practice. In: Jonassen, D. H.; Duffy, M.; Mahwah, N. (Ed.). Theoretical foundations of learning environments. Lawrence Erlbaum Associates, 25-55. [en línea] Disponible en: http://it.coe. uga.edu/itforum/paper28/paper28. html (consulta 2011, 10 de enero).

Bielaczyc, K.; Collins, A. (1999). Learning communities in classrooms: A reconceptualization of educational practices. In: Reigeluth, C. M. (Ed.). Instructional-design theories and models: Anew paradigm of instructional theory. New Jersey: Lawrence Erlbaum,
(269-292)

Cabero, J.; Llorente, M. C.; Puentes, A. (2010). La satisfacción de los estudiantes en red en la formación semipresencial. Comunicar, 35, (149-157).

Condi, R.; Livingston, K. (2007). Blending online learning with traditional approaches: changing practices. British Journal of Educational Technology, 38 (2), 337-348.

Coombe, K. (1999). Ethics and the learning community. In: Retallick, J.; Cocklin, B.; Coombe, K. (Eds.). Learning Communities in Education: Issues, strategies and contexts. London: Routledge, (86-104).

De Wever, B.; Schellens, T.; Valcke, M.; Van Keer, H. (2006). Content analysis schemes to analyze transcripts of online asynchronous discussion groups: A review. Computers y Education, 46, (628).

Downes, S. (1998). The future of online learning. [en línea] Disponible en: www. atl.ualberta.ca/downes/future (consulta 2011, 18 de enero).

Fahy, P. J.; Crawford, G.; Ally, M. (2001). Patterns of Interaction in a Computer Conference Transcript. International Review of Research in Open and Distance Learning, 1(4).

Garrison, D. R. (2007). Online community of inquiry review: Social, cognitive, and teaching presence issues. Journal of Asynchronous Learning Networks, 11 (1), (61-72).

Garrison, D. R.; Anderson, T.; Archer, W. (2000). Critical inquiry in a text-based environment: Computer conferencing 
in higher education. The Internet and Higher Education, 2, (1-19).

Garrison, D. R.; Anderson, T.; Archer, W. (2001). Critical thinking, cognitive presence, and computer conferencing in distance education. American Journal of Distance Education, 15 (1), (7-23).

Garrison, D. R.; Archer, W. (2003). A Community of Inquiry Framework for Online Learning. In: Moore, M. (Ed.), Handbook of Distance Education. New York: Erlbaum.

Garrison, D. R.; Cleveland-Innes, M.; Fung, T. (2004). Student role adjustment in online communities of inquiry: Model and instrument validation. Journal of Asynchronous Learning Networks, 8 (2), (61-74). [en línea] Disponible en: http://www.sloan-c.org/publications/ jaln/v8n2/pdf/v8n2 garrison.pdf (consulta 2011, 18 de enero).

Garrison, D. R.; Cleveland-Innes, M.; Koole, M.; Kappelman, J. (2006). Revisting methodological issues in the analysis of transcripts: Negotiated coding and reliability. The Internet and Higher Education, 9 (1), (1-8).

Graves, L. N. (1992). Cooperative learning communities: Context for a new vision of education and society. Journal of Education, 17 (2), (57-79).

Hiltz, S. R. (1998). Collaborative learning in asynchronous learning networks: Building learning communities. WEB98. Orlando. Florida. [en línea] Disponible en: http://eies.njit.edu/ hiltz/ collaborative learning in asynch.htm (consulta 2011, 1 de febrero).

Hinojo, F. J.; Aznar, I.; Cáceres, M. P. (2009). Percepciones del alumnado sobre el blended learning en la universidad. Comunicar 33, (165-174).

Jonassen, D.; Peck, K.; Wilson, B. (1999). Learning with technology: A constructivist perspective. Upper Saddle River, NJ: Merrill.
Ludwig-Hardman, S. (2003). Case study: Instructional design strategies that contribute to the development of online learning community. Denver: University of Colorado. Tesis doctoral inédita.

Mcmillan, D. W.; Chavis, D. M. (1986). Sense of community: A definition and theory. Journal of Community Psychology, 14 (1), (6-23).

Moore, M. G. (1993). Theory of transactional distance. In: Keegan, D.(Ed.). Theoretical principles of distance education. New York: Routledge, (22-38).

Retallick, J. (1999). Transforming schools into learning communities. In: Retallick, J.; Cocklin, B. y Coombe, K. (Eds.). Learning communities in education: Issues, strategies and contexts. London: Routledge, (107-130).

Rourke, L.; Anderson, T. (2004). Validity in Quantitative Content Analysis. Educational Technology Research and Development, 52 (1) (5-18).

Rovai, A. P. (2002). Building Sense of Community at a Distance. International Review of Research in Open and Distance Learning, 1 (3).

Salmerón, H.; Rodríguez, S.; Gutiérrez, C. (2010). Metodologías que optimizan la comunicación en entornos de aprendizaje virtual. Comunicar, 34, (163-171).

Shea, P.; Bidjerano, T. (2009). Community of inquiry as a theoretical framework to foster «epistemic engagement» and «cognitive presence» in online education. Computers y Education, 52 (3), (543553).

Tirado, R. (2002). Los entornos virtuales de aprendizaje. Bases para una didáctica del conocimiento. Granada: Grupo Editorial Universitario.

Tirado, R. (2003). Teleformación ocupacional. Granada: Grupo Editorial Universitario.

Tirado, R.; Guzmán, M. D.; Toscano, M. (2004). El proyecto Stopdrogas. La teleformación como espacio formativo 
intercultural interuniversitario. Comunicación y Pedagogía, 203, (5863).

Tirado, R.; Marín, I.; Lojo, B. (2008). Creando comunidades virtuales de aprendizaje en las prácticas curriculares. Pixel-Bit, 33, (133-153).

Tirado, R.; Aguaded, J. I.; Méndez, J. M. (2009). Interacciones en grupos de aprendizaje on line. Revista Iberoamericana de Educación, 48 (5).

Tirado, R.; Boza, A.; Guzmán, M. D. (2009). Efectos de las interacciones en la creación de comunidades virtuales de prácticas. Revista de Educación a Distancia, 21, (4-12).

Tirado, R.; Méndez, J. M. (2010). Creando comunidades virtuales de aprendizaje: análisis del progreso de las interacciones. Revista de Educación, 353, (297-328).

Tirado, R.; Hernando, A.; Aguaded, J. I. (2011). Aprendizaje cooperativo online a través de foros en un contexto universitario: un análisis del discurso y de las redes. Estudios sobre Educación, 20.

Tirado, R.; Pérez, A.; Aguaded, J. I. (2011).
Blended e-learning en universidades andaluzas. Aula Abierta, 39 (2), (47-58).

Wells, P. (1999). Different and equal: Fostering interdependence in a learning community. In: Retallick, J.; Cocklin, B.; Coombe,K.(Eds.).Learning communities in education: Issues, strategies and contexts. London: Routledge.

Wenger, E. (1998). Communities of practice: Learning, meaning and identity. Cambridge: Cambridge University Press.

Westheimer, J.; Kahne, J. (1993). Building school communities: An experiencebased model. Phi Delta Kappan 75 (4), (324-28).

Wilson, B. G.; Ludwing-Hardman, S.; Thornam, C. L.; Dunlap, J. C. (2004). Bounded Community: Designing and Facilitating Learning Communities in Formal Courses. International Review of Researchin OpenandDistance Learning, 3 (4). [en línea] Disponible en: www. irrold.org/index.php/article/view/204 (consulta 2010, 12 de diciembre).

Zhu, E. (2006). Interaction and cognitive

engagement: An analysis of four asynchronous online discussions. Instructional Science, $34,(451-480)$.

\section{PERFILES PROFESIONALES Y ACADÉMICOS DE LOS AUTORES}

Ramón Tirado-Morueta. Profesor Titular de la Universidad de Huelva (España), imparte docencia en la materia de Tecnología Educativa. Su carrera investigadora se ha organizado en torno a los efectos y variables moduladoras de la aplicación de las TIC en múltiples contextos educativos. Recientemente, ha publicado diversos artículos científicos en revistas de impacto internacional centrados en el análisis de procesos de aprendizaje cooperativo y comunidades de aprendizaje apoyados en el uso de los recursos tecnológicos, así como en el contraste de teorías sobre la influencia de factores externos e internos al docente en la integración de tecnologías de la información y comunicación en centros de educación primaria, secundaria y universidades. 
R. Tirado; Á. Hernando; I. AguAded

Comunidades de Aprendizaje a Través de Plataformas de Teleformación

\section{E-mail: rtirado@uhu.es}

Ángel Hernando-Gómez. Profesor Contratado Doctor. Universidad de Huelva. Profesor en el Departamento de Psicología Evolutiva y de la Educación de la Universidad de Huelva (España). Director del Servicio de Atención a la Comunidad Universitaria de esta Universidad. Académicamente es Doctor en Psicología, Maestro y Licenciado en Psicología y en Psicopedagogía. Director adjunto de la revista científica iberoamericana de comunicación y educación «Comunicar» y miembro del Consejo Editorial de «Apuntes de Psicología». Ha participado en múltiples actividades formativas y de investigación en el ámbito de la orientación escolar, la psicología de la educación, la intervención sobre conductas de riesgo y la promoción del desarrollo adolescente positivo. Es autor de muchas publicaciones donde aborda distintas temáticas, todas ellas relacionadas con el área de Psicología Evolutiva y de la Educación.

E-mail: angel.hernando@dpsi.uhu.es; direccion@sacu.uhu.es.

José Ignacio Aguaded Gómez. Catedrático de Universidad del Departamento de Educación de la Universidad de Huelva (España). Vicerrector de Tecnologías y Calidad de la Universidad de Huelva. Presidente del Grupo Comunicar, colectivo veterano en España en educomunicación, y Director de la Revista Científica Iberoamericana «Comunicar» (indexada en JCR). Es además Director del Grupo de Investigación "Ágora», responsable de múltiples proyectos de investigación nacionales e internacionales y la dirección de numerosas tesis doctorales. Es Director del Máster Internacional Interuniversitario de Comunicación y Educación Audiovisual (UNIA/UHU).

E-mail: vicerrector.tecnologias@uhu.es

\section{DIRECCIÓN DE LOS AUTORES:}

Universidad de Huelva Rectorado.

Campus Cantero Cuadrado, s/n. 21071 Huelva (España). Tfno: 0o-34-959-218295; Fax: 0o-34-959-248380.

Fecha de recepción del artículo: 19/02/2011

Fecha de aceptación del artículo: 14/06/2011

\section{Como citar este artículo:}

Tirado-Morueta, R.; Hernando-Gómez, A.; Aguaded, I. (2011). Comunidades 